\title{
RISCO À INUNDAÇÃO NO MÉDIO CURSO DO RIO URUGUAI: ESTUDO DE CASO NO MUNICÍPIO DE SÃO BORJA - RS
}

\section{FLOOD RISK IN THE MIDDLE URUGUAI RIVER: CASE STUDY OF THE SÃO BORJA MUNICIPALITY - RS}

Eléia Righi
Universidade Federal do Rio Grande do Sul - Mestre em Geografia (POSGEA) - Rua Jacinto Gomes, 344, ap 01, Bairro
Santana, POA, RS, CEP: 90040-270 - e-mail: leiarighi@yahoo.com.br; eleia@profill.com.br

Luis Eduardo de Souza Robaina

Universidade Federal de Santa Maria - Depto de Geociências - Prédio 17 - Depto de Geociências, CEP 97105-900, Camobi - Santa Maria - RS - e-mail: lesrobaina@yahoo.com.br

\section{Informações sobre o Artigo}

Data de Recebimento:

19/09/2011

Data de Aprovação:

$03 / 10 / 2012$

\section{Palavras-chave:}

Inundação Gradual, Rio Uruguai, Zoneamento de Risco.

\section{Keywords:}

Flood, Uruguay River, Risk Zoning.

\begin{abstract}
Resumo
Diante de tantos prejuízos oriundos das inundações, observam-se poucos trabalhos visando à espacialização e a análise desses eventos no Brasil e no Rio Grande do Sul. Neste sentido, esse trabalho tem por objetivo realizar o Zoneamento de Risco à Inundação no município de São Borja, localizado na margem esquerda do rio Uruguai no Estado do Rio Grande do Sul. Foram levantadas as ocorrências de inundações no município, verificando as situações de emergência e calamidade pública entre 1980 a 2009. Posteriormente foi determinada a suscetibilidade, o perigo dividido em três graus (alto $<10$ anos de tempo de retorno (TR), médio entre 10 e 30 TR e baixo $>30$ anos de TR), a vulnerabilidade através do cruzamento das características de infraestruturas e número médio de habitantes por residências, e o risco a partir da analise integrada dos graus de perigo e classes de vulnerabilidade. Em relação à quantidade de eventos, verificou-se 38 registros de inundações. As áreas suscetíveis correspondem a $11 \%$ da área urbana. Em relação ao perigo, nas três faixas (alto, médio e baixo) foi verificado um número significativo de edificações. $\mathrm{Na}$ análise da vulnerabilidade, foram encontradas construções com usos residenciais, comerciais e industriais, com padrão estrutural alto, médio e baixo. O mapa de risco de inundação foi elaborado caracterizando três níveis: zona de alto risco, médio risco e baixo risco. Assim, com essas análises, pretendemos contribuir com uma fonte teórico-metodológica como também com um trabalho técnico para o município, pois serve de base para estudos de maior detalhe que desenvolvam um cadastramento do risco, indicando ações para cada caso.
\end{abstract}

\begin{abstract}
Even with so many losses from the floods, there are few works and the spatial analyses of these events in Brazil and Rio Grande do Sul. In this sense, this work aimed to perform a Flood Risk Zoning of the São Borja city, located on the left bank of the Uruguay River in the Rio Grande do Sul State. We surveyed the flood events in this city, checking emergency
\end{abstract}


and public disaster from 1980 to 2009. Further, we accomplished the flood susceptibility analysis, where the grades of hazard were divided into three grades (high $<10$ years of return time (RT), medium between 10 and 30 and low RT $>30$ years). The vulnerability grade was built through the intersection of the characteristics of infrastructure and the mean number of inhabitants per residence. The risk grades were generated from the integrated data analysis of the degrees of hazard and vulnerability grades. Regarding the number of events, there were 38 records of flooding. The areas susceptibility corresponds to $11 \%$ of the urban area. Regarding the hazard, for the three bands (high, medium and low) the significant numbers of buildings were found. In vulnerability analysis, buildings with residential, commercial and industrial structural pattern with high, medium and low were found. The flood risk map was prepared featuring three levels: in the high risk, medium risk and low risk. Thus, this study produced the technical and theoretic-methodological insights to the flood management in this city, and it serves as a basis for further detailed studies to develop a risk register, indicating actions to each case.

\section{Introdução}

Muitos municípios do Rio Grande do Sul, principalmente os que margeiam o rio Uruguai, apresentam registros de processos de inundações. O baixo conhecimento técnico, sobre a dinâmica fluvial e atmosférica, aliada à falta de políticas de restrições à ocupação de áreas de perigo, deixa a população vulnerável à inundações.

Mesmo assim, diante de tantos prejuízos oriundos das inundações, não se encontram muitos trabalhos visando a espacialização e a análise desses eventos nos municípios em baixo curso do rio Uruguai.

Righi (2011), bem como Reckziegel e Robaina (2007) e Reckziegel (2007), apontam que muitos municípios que margeiam o rio Uruguai possuem mais de 38 ocorrências registradas de desastre por município, entre 1980 a 2005. Muitas cidades ribeirinhas desse grande rio já perderam centenas de moradias que ficaram submersas, assim como houve destruição de lavouras, estradas e pontes.

Segundo Alcântara Ayala (2002), a ocorrência de inundações é ligada não somente a suscetibilidade dos locais às características geoambientais, mas também a vulnerabilidade do sistema da sociedade, isto é, sistema econômico-socialpolítico-cultural.

Quando ocorre a combinação entre incerteza, exposição ao perigo, perda e prejuízos materiais, econômicos e humanos em função de processos de inundação pode-se denominar risco (CASTRO, OLIVEIRA PEIXOTO e PIRES do RIO, 2005). A existência de um risco só se constitui quando há a valorização de algum bem, material ou imaterial, pois não há risco sem a noção de que se pode perder alguma coisa.

Assim, o presente trabalho tem por objetivo apresentar o zoneamento de risco à inundação no município de São Borja localizado na margem esquerda do rio Uruguai no RS (Figura 1). O zoneamento de risco destaca-se como medida não estrutural essencial para o gerenciamento auxiliando no estabelecimento de medidas que minimizem perdas e danos.

Além disso, os mapas subsidiam a reorganização do espaço urbano, com o estabelecimento de novas diretrizes de ocupação nas cidades.
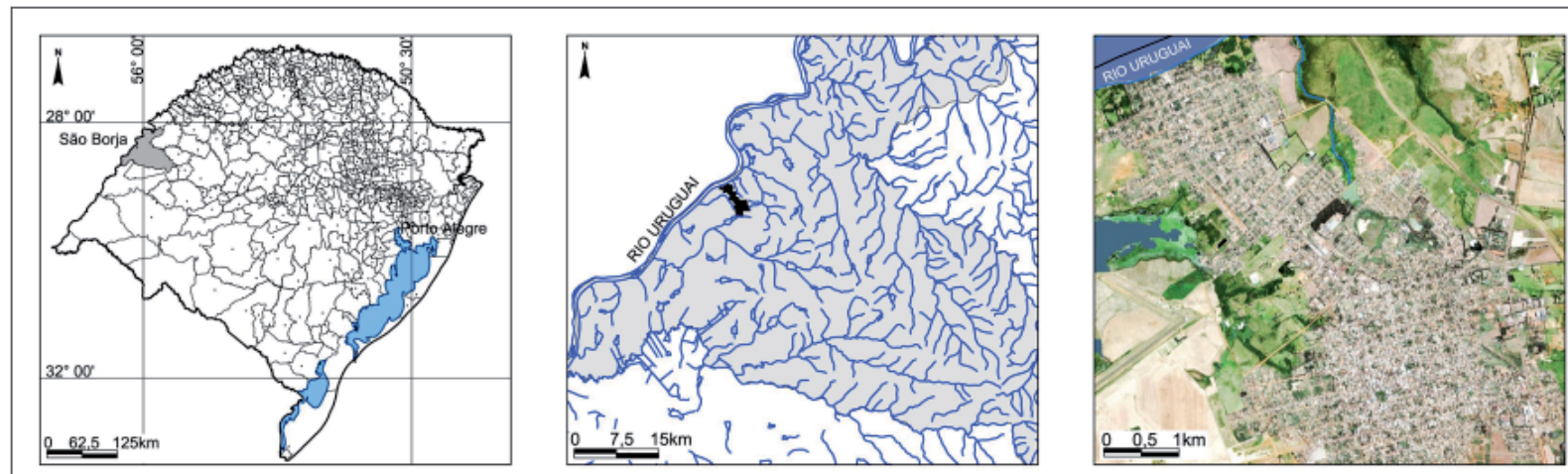

\begin{tabular}{|c|c|c|}
\hline 00 & & JUN / 2011 \\
\hline Revisato & Discriminaçấ & Data \\
\hline & PROGRAMA DE POS-GRADUACGAO EM GEOGRAFIA & \\
\hline UFRंGS & $\begin{array}{l}\text { RISCO A INUNDAÇĀO NO MÉDIO CURSO DO RIO URUGUAL: } \\
\text { ESTUDO DE CASO NO MUNICIPIO DE SÃO BORJA - RS } \\
\text { RIO GRANDE DO SUL. }\end{array}$ & \\
\hline \multirow{2}{*}{$\begin{array}{l}\text { Datum SAD } 69 \\
\text { Projechlo UTM } \\
\text { Fuso } 21\end{array}$} & \multicolumn{2}{|l|}{ Asporito } \\
\hline & \multicolumn{2}{|l|}{ Situação e Localização } \\
\hline
\end{tabular}

Figura 1 - Mapa de situação e localização de São Borja 


\section{Metodologia}

Visando a melhor compreensão dos procedimentos metodológicos adotados neste trabalho, optou-se por uma estruturação em duas etapas, de acordo com o nível de detalhamento dos mapeamentos.

\section{Etapa I: Levantamento de Materiais}

O levantamento dos dados referentes à ocorrência de inundações graduais foi embasado no trabalho realizado por Reckziegel (2007), a qual realizou levantamentos entre 1980 a 2005, utilizando duas fontes de dados para os 26 anos analisados: Diário Oficial e informações na imprensa escrita.

Os dados legais referem-se aos decretos de Situação de Emergência e de Estado de Calamidade Pública, tendo sido levantados junto à Defesa Civil do Estado no Rio Grande do Sul e nas publicações do Diário Oficial do Estado. O veículo de imprensa escolhido foi o jornal Zero Hora, no qual foram levantadas as reportagens que continham alguma notícia a respeito da ocorrência de inundações.

Reckziegel (2007) explica que os dados levantados junto ao banco de dados da Defesa Civil do Estado correspondem aos eventos ocorridos entre os anos de $1991 \mathrm{e}$ 2005. Como a Defesa Civil não dispõem de informações sobre a ocorrência de eventos adversos e a homologação de decretos de Situação de Emergência e de Estado de Calamidade Pública anteriores ao ano de 1991, o levantamento dos decretos homologados no período de 1980 a 1990 foi realizado no Diário Oficial e na Coletânea de Leis e Decretos, disponíveis na Biblioteca do Tribunal de Justiça do Estado.

A coleta de informações junto à imprensa foi realizada com o objetivo de complementar os dados referentes à ocorrência de inundações. A escolha do jornal Zero Hora como fonte de informação deve-se a maior facilidade de obtenção dos dados, devido a boa organização de seu acervo.

As ocorrências entre 2006 e 2009 foram levantadas junto ao banco de dados da Defesa Civil do Estado que se encontra no site da mesma (www.defesacivil.rs.gov.br).

O material cartográfico utilizado consiste na base cartográfica da Diretoria do Serviço Geográfico do Exército na escala 1:50.000 e na escala 1:250.000 de todo o Rio Grande do Sul, e nas curvas de nível de um metro de equidistância das áreas urbanas.

Os recortes de imagens QuickBird derivadas do software Google Earth ${ }^{\mathrm{TM}}$ foram baixadas do Google Earth-Pró 2010, e as imagens LANDSAT foram disponibilizadas pelo INPE (www.inpe.br), as selecionadas para serem classificadas na ocorrência de inundações são: WRS 225/080 de 08/06/1990, WRS 2240/080 de 18/09/1989 e WRS 224/079 de 18/09/1989.

As imagens SRTM foram adquiridas gratuitamente, através da EMBRAPA (www.embrapa.br).

Os dados fluviométricos foram obtidos na Agência Nacional das Águas - ANA, disponíveis no site da ANA (www.ana.gov.br), onde foram levantados dados diários de vazão e cotas. A série histórica da estação São Borja (Código 75780000; Altitude 50m; Latitude -28:37:28, Longitude $-56: 2: 13$ ), localizada neste município, apresenta dados desde 1940.

\section{Etapa II: Processamento e Relação entre os Dados}

A delimitação e análise das áreas suscetíveis à inundação foram realizadas a partir da integração de parâmetros relativos ao relevo (declividade $<2 \%$ ), rede de drenagem, unidades geomorfológicas (RADAM/IBGE, 2003) e classificação de imagens de satélite.

O mapeamento das áreas de perigo levou em consideração as áreas urbanas ocupadas dentro das áreas suscetíveis a inundação, delimitadas na fase anterior. A partir da linha de delimitação das áreas de perigo, realizou-se a classificação de graus de perigo, através do tempo de retorno das inundações, quantidade de eventos registrados e trabalhos de campo.

A partir da linha de delimitação das áreas de perigo, realizou-se a classificação de graus de perigo, através do tempo de retorno das inundações, quantidade de eventos e trabalhos de campo.

Nos trabalhos de campo obteve-se informações específicas sobre as inundações ocorridas, verificando as cotas altimétricas atingidas; realizou-se entrevistas com moradores locais buscando informações sobre os eventos; percorreu-se toda a área urbana com perigo dos municípios para analisar a paisagem e identificar possíveis fatores de influência sobre as inundações.

O cálculo do tempo de retorno se baseou em Christofoletti (1981), que compreende a seleção do evento de maior magnitude (vazão) em cada ano da série histórica, o ordenamento dos eventos conforme a sequencia de magnitude (ranking) dos valores mais elevados para os menores e a aplicação da seguinte equação:

$$
\mathrm{TR}=(\mathrm{N}+1) / \mathrm{M}
$$

onde TR é o tempo de retorno; $\mathrm{N}$ é o número total de eventos considerados na série; e $\mathrm{M}$ é o número de ordem (ranking) que representa a posição da inundação na escala organizada.

O método para modelagem tridimensional do relevo foi desenvolvido a partir das projeções de inundações, 
como o uso da computação gráfica tridimensional. O primeiro passo foi a organização das curvas topográficas de 1 para 1 metro das cidades com o software AutoCAD 2010.

A seguir foi feito o ajuste dos objetos para a projeção tridimensional, a partir das curvas. Com o desenho ajustado, criou-se uma rotina LISP (linguagem de programação do software AutoCAD 2010). Esta rotina cria uma grade de pontos em uma área pré-determinada e a partir daí ela lê as cotas de cada ponto, este processo é feito quatro vezes pelo programa.

Assim, foram individualizados 3 graus de perigo, considerando que o grau de alto perigo possui TR menor de 10 anos e representa a maioria dos eventos ocorridos, sendo maior que seis. A faixa de perigo médio possui entre seis a dois eventos, com um TR entre 10 e 30 anos. A terceira faixa, considerada de perigo baixo representa os eventos mais excepcionais, ocorrendo uma ou duas vezes na série histórica analisada, possuindo tempo de retorno acima de 30 anos.

A partir da definição da área de perigo analisouse a vulnerabilidade, determinada pelas edificações conforme o padrão urbano, enquanto sua apresentação material visível aos olhos do observador, infraestrutura e o ordenamento da ocupação.

Dessa forma, o padrão alto corresponde àquelas edificações que foram construídas com técnicas de engenharia adequadas segundo as normas construtivas e apresentam material de boa qualidade em sua construção (edificações de alvenaria). O padrão urbano médio corresponde às casas de madeira que possuem uma base de alvenaria, normalmente as casas de madeiras são mais rápidas e fáceis para a construção, mais baratas e não necessitam de uma mão de obra especializada, e, a base de alvenaria faz com que a residência suporte mais o impacto das águas.

Já o baixo padrão construtivo está vinculado às habitações que geralmente são auto construídas por seus moradores e apresentam variados materiais em sua estrutura. Foram também consideradas algumas casas de madeiras que não possuem uma base de alvenaria, por possuírem uma fragilidade maior quando na ocorrência de inundações.

Em relação à infraestrutura foram analisadas as malhas viárias, obras de engenharia para contenção de inundações, rede de drenagem pluvial e redes de água. Essas variáveis foram verificadas nos trabalhos de campo, imagens QuickBird e informações obtidas nos órgãos públicos. Assim, os locais que possuíam uma boa malha viária, boas condições de drenagem urbana e obras de contenção a inundações foram classificadas com baixo grau de vulnerabilidade.
Os locais com médias condições, ou seja, pontos que possuíam algum desses itens em condições razoáveis foram classificados com média vulnerabilidade e os locais com condições ruins foram classificadas com alto grau de vulnerabilidade.

Além disso, foi verificada a quantidade de habitantes por setores censitários a partir dos dados de população do Instituto Brasileiro de Geografia e Estatística (IBGE): Resultados do Universo do Censo Demográfico 2000 e 2007. Estes dados foram validados e analisados em trabalhos de campo.

As classes de vulnerabilidade são obtidas a partir destes levantamentos, indicado no Quadro 1.

Posteriormente realizou-se o zoneamento do risco a inundações a partir da analise integrada dos graus de perigo e classes de vulnerabilidade (Quadro 2).

\section{Quadro 1 - Matriz para o cálculo das classes de vulnerabilidade.}

\begin{tabular}{ccccc}
\multicolumn{2}{c}{ Variáveis Sociais } & \multicolumn{3}{c}{ Número de habitantes afetados } \\
& & $>36$ & $16-35$ & $5-15$ \\
\hline $\begin{array}{c}\text { Condições de } \\
\text { infraestrutura }\end{array}$ & 1 - Baixo & 1 (alto) & 2 (alto) & 3 (médio) \\
$\begin{array}{c}\text { e padrão das } \\
\text { edificações }\end{array}$ & 3 - Médio & 2 (alto) & 4 (médio) & 6 (baixo) \\
& & 3 (médio) & 6 (baixo) & 9 (baixo) \\
\hline
\end{tabular}

\section{Quadro 2 - Matriz para o cálculo do zoneamento de} risco.

\begin{tabular}{|c|c|c|c|c|}
\hline \multirow{2}{*}{\multicolumn{2}{|c|}{ Perigo $x$ Vulnerabilidade }} & \multicolumn{3}{|c|}{ Perigo } \\
\hline & & 1 - Alto & 2-Médio & 3 - Baixo \\
\hline \multirow{3}{*}{ Vulnerabilidade } & 1 - Alta & 1 (alto) & 2 (alto) & 3 (médio) \\
\hline & 2 - Média & 2 (alto) & 4 (médio) & 6 (baixo) \\
\hline & 3 - Baixa & 3 (médio) & 6 (baixo) & 9 (baixo) \\
\hline
\end{tabular}

\section{Resultados}

As análises das características geomorfológicas permitiram a identificação das áreas suscetíveis à inundação. Os dados mostram que essas áreas, na parte central do município de São Borja, chegam, aproximadamente, a 4000 metros de extensão a partir da calha do rio Uruguai. $\mathrm{Na}$ área urbana, que corresponde a porção que interessa ao trabalho, a suscetibilidade a inundações aumenta na parte oeste e norte, que estão as margens do rio Uruguai e do arroio Itachere (Figura 2).

Os dados que indicam suscetibilidade na área urbana, somados com o inventário da ocorrência de eventos (ao total 38, sendo decretadas 15 situações de 
emergência e uma de calamidade pública) determinam as áreas de perigo. As áreas de perigo ocupam uma parcela significativa do município, cerca de $11 \%$ da área urbana (Figura 2).

O grau de perigo caracterizado em três níveis: Perigo I, Perigo II e Perigo III indicam o tempo de retorno dos eventos (Figura 3). Na zona de alto perigo (Perigo I), que possui tempo de retorno até 10 anos, encontram-se muitas edificações, um total de 337. Entretanto, como acontece no bairro Passo onde está localizado o Cais do Porto muitas edificações são usadas com fins comerciais para bares e restaurantes.

$\mathrm{Na}$ parte norte da área urbana, no bairro Itachere encontram-se muitas residências nessa faixa de perigo, localizando-se ao longo do arroio Itachere, o qual possui nas suas nascentes um açude. Nesta área as inundações da área urbana podem ser explicadas pelas declividades baixas e posição topográfica muito próxima ao arroio Itachere, que sofre bloqueios das suas águas pelo aumento do nível do rio Uruguai.

As áreas caracterizadas de médio perigo, com tempo de retorno de 10 a 30 anos, possuem um total de 240 edificações, possuindo usos residenciais, bem como industriais, institucionais e comercias.

A zona de baixo perigo - Perigo III - possui pequena probabilidade de ocorrência de inundações, somente será atingida em anos excepcionais. Apesar da menor probabilidade de ocorrência de eventos o número de edificações é significativo, um total de 1075.

Um dos parâmetros para determinar a vulnerabilidade são as características dos arruamentos, pois constitui um indicador do nível das estruturas e grau de organização do sistema social. Assim, foi verificado que cerca de $80 \mathrm{~km}$ das vias públicas não possuem pavimentação, sendo apenas encascalhadas. Dos $120 \mathrm{~km}$ pavimentados, $40 \mathrm{~km}$ são asfaltados no centro da cidade e $80 \mathrm{~km}$ pavimentados com pedras imbricadas a guisa de paralelepípedos.

Os arruamentos inter-bairros são constituídos de revestimentos muitas vezes degradados ou inexistentes, em alguns trechos os veículos trafegam diretamente sobre o que se pode considerar como revestimento primário e solo compactado. Os passeios observados não possuem geometria definida e são formados por materiais diversos, predominando o solo compactado.

São Borja, assim como observado por Costa et al. (2009) no município de Caratinga-MG, apresenta um padrão urbano diferente daquele que encontramos normalmente em áreas de perigo aos processos fluviais, as construções possuem em geral um bom padrão construtivo, a maioria das moradias possui excelentes acaba- mentos e boas condições de conservação. Estes bairros apresentam alto grau de ocupação e pouca presença de ocupações irregulares. Isso, provavelmente, se associa com a forte relação da cidade com o rio desde sua fundação e as trocas comerciais com a cidade Argentina de São Tomé.

As edificações com padrão urbano médio e baixo (habitações alto-construídas com variados materiais em sua estrutura) foram localizadas principalmente no bairro Passo.

O uso do solo é predominante residencial unifamiliar. Algumas residências possuem uso misto para fins comerciais ou de serviços, voltadas à complementação do orçamento familiar. Ainda encontram-se no município muitos pescadores, que moram próximo ao rio Uruguai.

Com os dados obtidos determinou-se o zoneamento da vulnerabilidade mostrado no mapa de vulnerabilidade, da Figura 4.

A classe de baixa vulnerabilidade está presente ao Norte da área urbana, junto ao arroio Itachere possuindo em torno de 29 residências com alto padrão estrutural, 18 torres de silos para armazenamento de grãos e cinco edificações com outros usos. O número de pessoas neste local é em torno de 100.

Nas proximidades do rio Uruguai esta classe possui um total de 46 edificações comerciais, ao meio destas edificações encontram-se algumas residências com alto padrão estrutural.

A classe de média vulnerabilidade também predomina ao norte da área urbana e nas proximidades do rio Uruguai. O número de edificações é em torno de 1155 , sendo somente 63 para outros usos (não residenciais), assim o número de pessoas também é alto em torno de 3800 pessoas.

A classe de alta vulnerabilidade localiza-se próximo ao Caís do Porto, com um padrão construtivo médio e baixo, um sistema de esgoto inadequado bem como de ruas e imobiliário urbano. Este local possui um total de 238 residências, consequentemente um número aproximado de 800 pessoas. Na parte norte da área urbana encontra-se 138 edificações, com aproximadamente 480 pessoas.

O risco pode ser analisado como uma categoria associada à priori como uma área passível de ser atingida por processos naturais e/ou induzidos que causem efeito adverso. As pessoas que habitam essas áreas estão sujeitas a danos à integridade física, perdas materiais e patrimoniais (BRASIL/IPT, 2004).

O mapa de risco de inundação foi elaborado caracterizando três níveis: Risco Baixo, Risco Médio e Risco Alto (Figura 5). As áreas caracterizadas de baixo risco 
(tempo de retorno acima de 30 anos) possuem predominantemente edificações com médio e alto padrão construtivo. Entretanto, deve-se considerar que se em algum evento de inundação ocorrer e todas as 902 residências forem atingidas sofreram algum tipo de dano, pelo menos, 3000 pessoas.

Nesta faixa foi identificado um total de 100 edificações para fins industriais, institucionais, comercias e lazer, como também 18 torres de silos, utilizadas para armazenamento de grãos.

O médio risco ocorre quando os dois parâmetros de análise são médios; onde o perigo de ocorrer um evento é alto, mas a vulnerabilidade da população é baixa. Nesta zona, encontram-se um total de 239 edificações, sendo caracterizadas com médio e alto padrão urbano usadas com fins residências e comerciais (14 edificações somente).

A zona de alto risco é definida por um tempo de retorno inferior a 10 anos e as ocupações possuem predomínio de baixo e médio padrão construtivo. Nela encontram-se um total de 410 edificações.

Se todas essas residências fossem atingidas em um evento teriam que ser removidas em torno de 1000 pesso- as. Assim, explica-se a quantidade de pessoas desabrigadas/atingidas nos eventos registrados no município.

Para as edificações de baixo padrão estrutural as inundações são bem destrutivas, o efeito da duração da inundação sobre as residências quanto mais prolongadas forem pode lhes causar danos irreparáveis. Se a inundação for da ordem de dias, exigirá que abandonem as casas.

A Prefeitura de São Borja tem buscado um gerenciamento das áreas de risco. Uma nova meta para o novo Plano Diretor é a desocupação abaixo da cota topográfica de 63 metros (faixa de alto risco, onde se encontram a maior quantidade de residências de baixo padrão estrutural), e que seja vedado o parcelamento do solo, para fins urbanos.

Ação importante realizada pela Prefeitura foi a retirada de muitas famílias nos últimos anos em situação de alto risco sendo recolocadas no bairro Itachere. Como o bairro Passo é o primeiro a ser atingido pelas inundações do rio Uruguai, uma outra iniciativa foi a construção de uma bacia de detenção. A Prefeitura Municipal está propondo também a criação de uma nova área de preservação, este ainda é um projeto, mas que ajudará a prefeitura a controlar a ocupação dessas áreas.

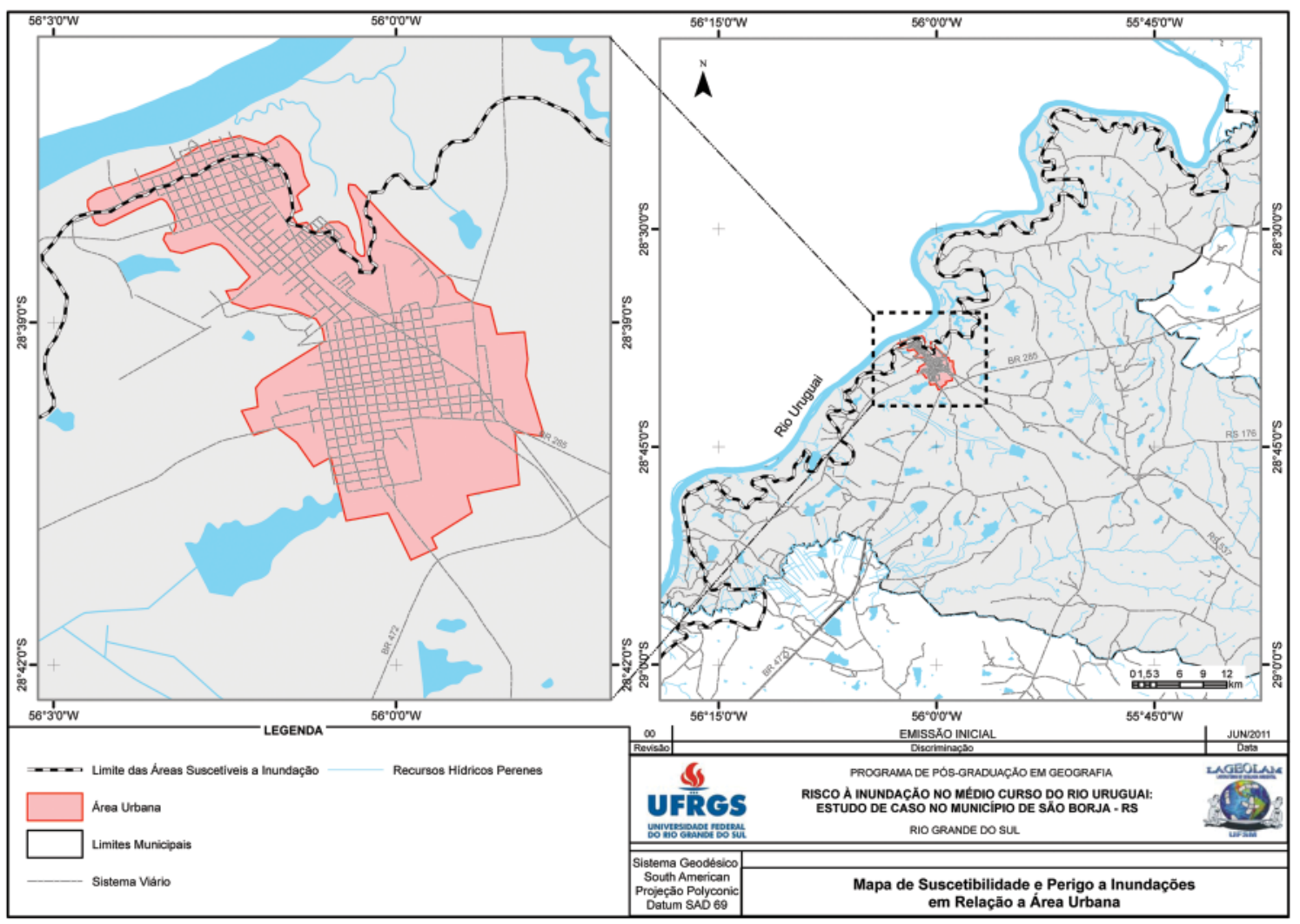

Figura 2 - Mapa de Susceptibilidade e Perigo a Inundações em Relação à Área Urbana 


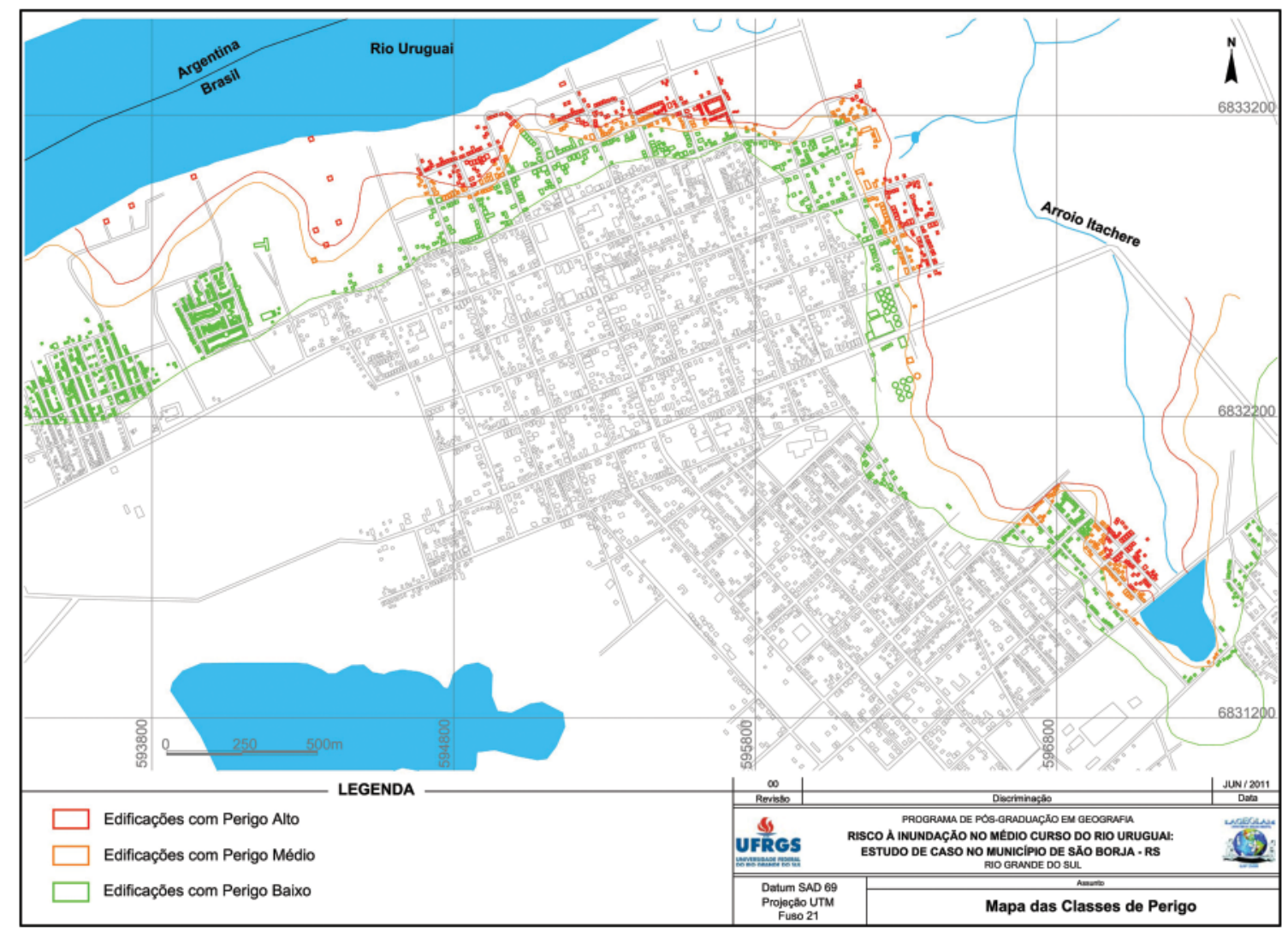

Figura 3 - Mapa das Classes de Perigo

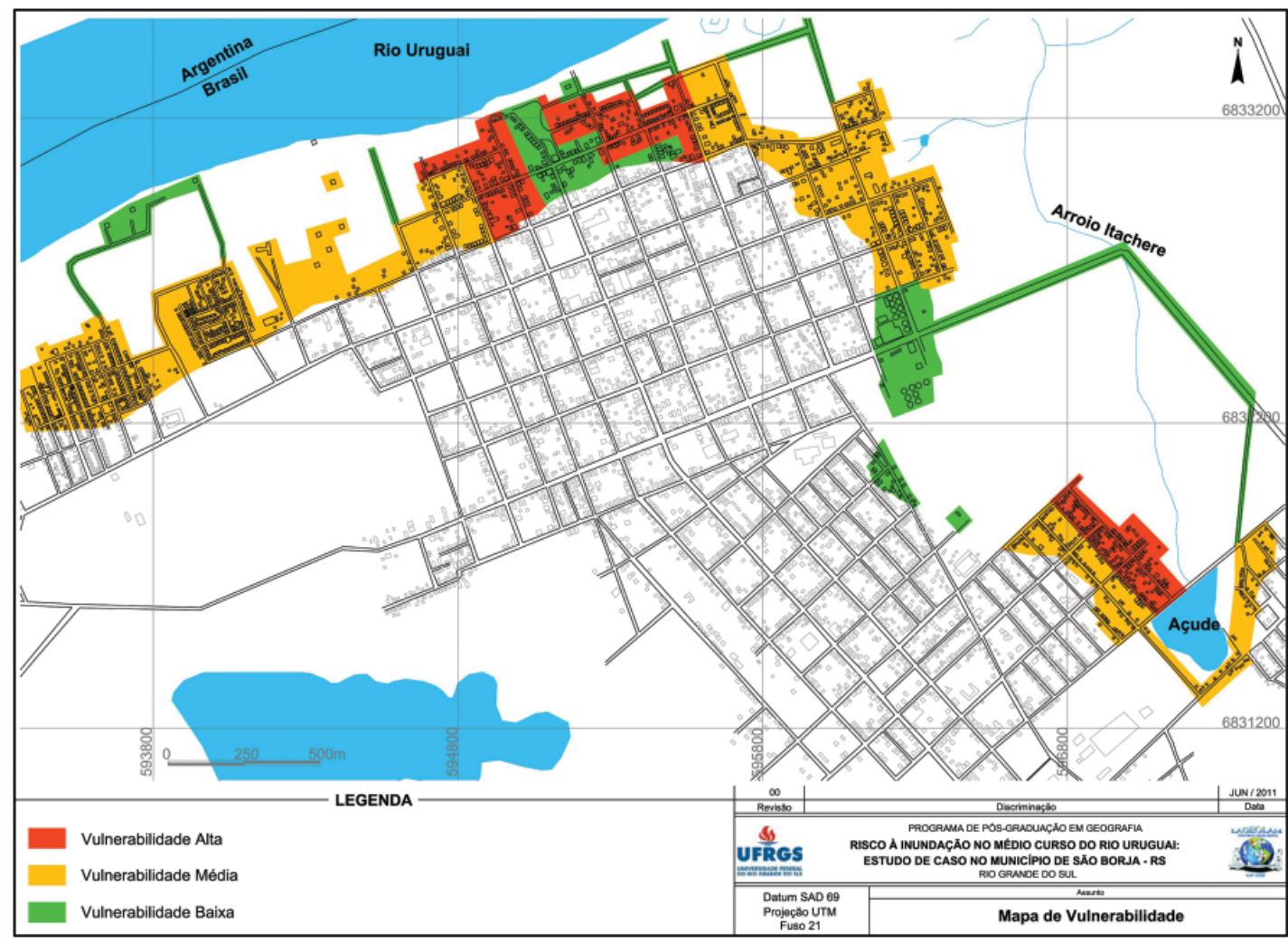

Figura 4 - Mapa de Vulnerabilidade 


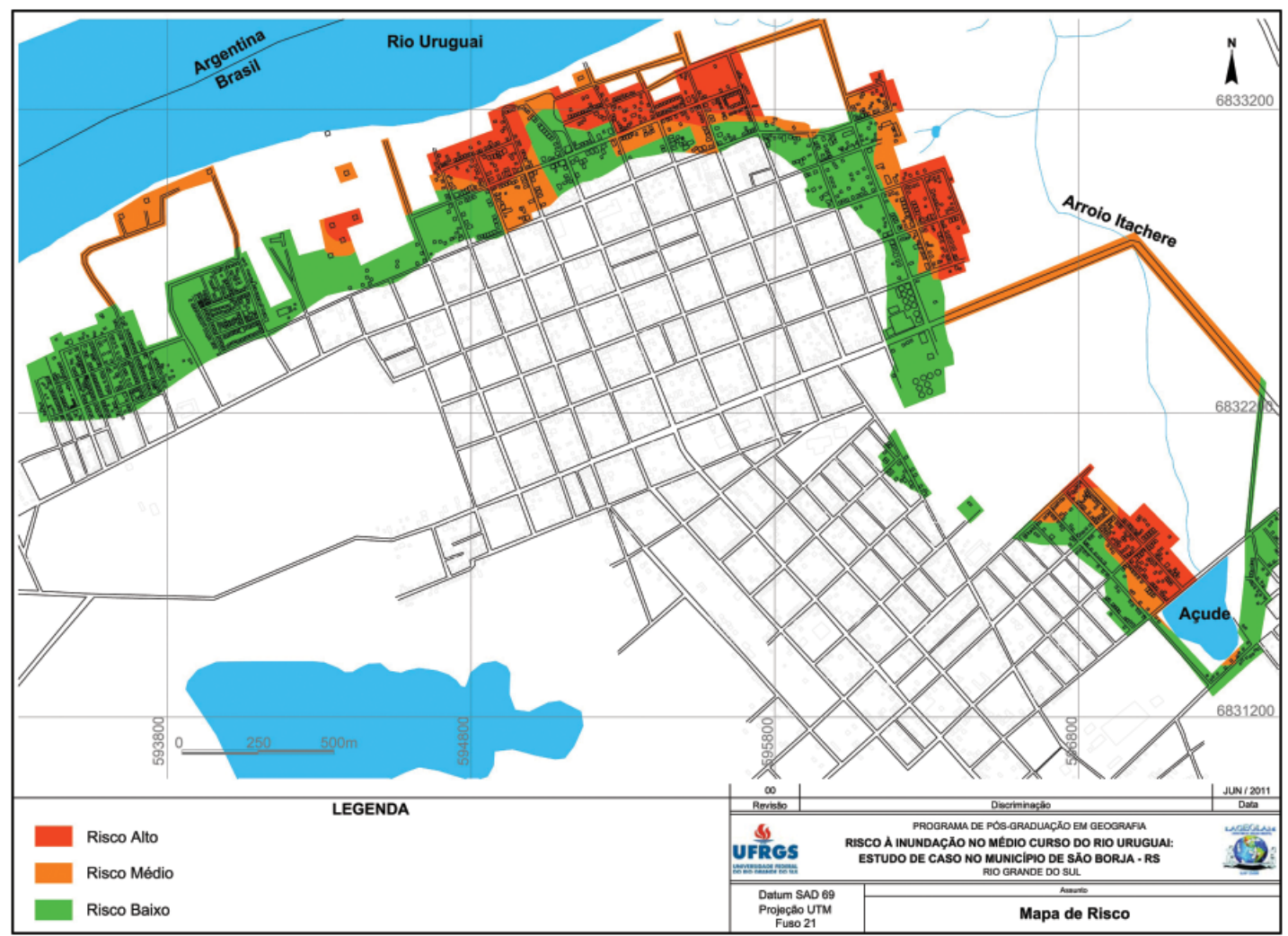

Figura 5 - Mapa de Risco

\section{Considerações finais}

O conhecimento das áreas de perigo, vulnerabilidade e risco de inundações, é indiscutivelmente fundamental ao desenvolvimento socioeconômico de São Borja.

Os resultados mostram a situação e localização da população residente nas áreas de risco, podendo ser geradas e aplicadas metodologias que venham a melhorar a qualidade de vida dessas pessoas, como também possuir um melhor convívio com as inundações, reduzindo os danos causados por esses eventos.

\section{Referências bibliográficas}

ALCÁNTARA AYALA, I. Geomorphology, natural hazard, vulnerability and prevention of natural disasters developing countries. Geomorphology, v. 47, p.107-124, 2002.

BRASIL. MINISTÉRIO DAS CIDADES/IPT. Treinamento de Técnicos Municipais para o Mapeamento e Gerenciamento de Áreas Urbanas com Risco de Escorregamentos, Enchentes e Inundações. Apostila de Treinamento. 2004, 73p.

CASTRO, C. M.; OLIVEIRA PEIXOTO, M. N.; PIRES DO RIO, G. A. Riscos Ambientais e Geografia: Conceituações, Abordagens e Escalas. Anuário do Instituto de Geociências UFRJ. ISSN 0101-9759, Vol. 28-2 / 2005 p. 11-30, 2005.
CHRISTOFOLETTI, A. Geomorfologia Fluvial. São Paulo: Edgard Blucher, 1981.

COSTA, M. das G. A. et al. Avaliação de áreas de risco à inundação no perímetro urbano de Caratinga - MG através do uso de dados altimétricos provenientes do sensor aerotransportado LIDAR. In: Anais do XIII Simpósio Brasileiro de Geografia Física Aplicada, Universidade Federal de Viçosa, Viçosa/MG, 2009.

RECKZIEGEL, B. W. Levantamento dos Desastres Desencadeados por Eventos Naturais Adversos no Estado do Rio Grande do Sul no Período de 1980 a 2005. 2007. V1. Dissertação (Mestrado em Geografia) - Universidade Federal de Santa Maria, Santa Maria, 2007.

RECKZIEGEL, B. W; ROBAINA, L. E. S. Desastres Naturais no Estado do Rio Grande do Sul no Período de 1980 a 2005: Enchentes e Enxurradas. 2 SIBRADEN. Anais..., São Paulo: Santos, 2007.

RIGHI, E. Risco a Inundação em Médio Curso do Rio Uruguai: um Estudo de Caso nos Municípios de Porto Lucena e São Borja. Dissertação (Mestrado em Geografia). Porto Alegre: UFRGSPOSGEA, 2011. 